\title{
First results from an extended freed-isobar analysis at COMPASS
}

\author{
Fabian Michael Krinner*† \\ Institute for Hadronic Structure and Fundamental Symmetries \\ E-mail: fabian-krinner@mytum.de
}

\begin{abstract}
One of the goals of the COMPASS experiment is the precision study of light-meson spectroscopy with data for various final states. With $46 \times 10^{6}$ exclusive events, the process $\pi^{-} p \rightarrow \pi^{-} \pi^{+} \pi^{-} p$ constitutes the flagship channel.

Based on this data set, the results of an extensive partial-wave analysis (PWA), using a total of 88 partial waves in the model, were published. Along with it, results of a first study of the so-called freed-isobar method were shown. Here, the fixed amplitudes for appearing $\pi^{+} \pi^{-}$intermediate states used in the conventional analysis were replaced by sets of piecewise constant functions to extract the amplitudes of the $\pi^{+} \pi^{-}$subsystems directly from the data. In this first study, this was done for three $\pi$ partial waves with $J^{P C}=0^{++}$intermediate $\pi^{+} \pi^{-}$states.

The promising results inspired further extension of this method, by also freeing intermediate $\pi^{+} \pi^{-}$states with $J^{P C}=1^{--}$and $2^{++}$. With this extension of the set of freed waves, mathematical ambiguities in the model arise due to the much higher flexibility of the model. We will present first results of these extended studies on COMPASS data along with methods to resolve the arising ambiguities.
\end{abstract}

XVII International Conference on Hadron Spectroscopy and Structure

25-29 September, 2017

University of Salamanca, Salamanca, Spain

\footnotetext{
${ }^{*}$ Speaker.

${ }^{\dagger}$ For the COMPASS collaboration
} 


\section{Freed-isobar partial wave analysis}

Due to the large data set collected by COMPASS, more elaborate analysis methods become applicable. One of these methods is the so called freed-isobar PWA. This approach is an extension of the conventional three-body PWA, that relies on the isobar model. In this model, the process is modeled as a two step decay of an intermediary state $X^{-}$into one of the final state $\pi^{-}$and another intermediary state $\xi$, the isobar. The isobar subsequently decays into $\pi^{+} \pi^{-}$. Within this model the intensity distribution $\mathscr{I}(\vec{\tau})$ for the $3 \pi$ final state is modeled as coherent superposition of waves with given angular momentum quantum numbers and known isobar resonances. These quantum numbers are the spin $J$ and eigenvalues under parity and generalized charge conjugation $-P$ and $C$ - the spin projection $M$, and reflectivity $\varepsilon$ of $X^{-}$, as well as the analogous quantum numbers $J_{\xi}^{P C}$ of the isobar. With these quantum numbers defining a partial wave, we can write the intensity distribution:

$$
\mathscr{I}(\vec{\tau})=\mid\left.\sum_{i \in \text { waves }} \mathscr{T}_{i}\left[\psi_{i}(\vec{\tau}) \Delta_{i}\left(m_{2 \pi}\right)+\text { Bose symm. }\right]\right|^{2},
$$

as a function of the phase space variables $\vec{\tau}$. The complex-valued production amplitudes $\mathscr{T}_{i}$ describe the intensities and relative phases of the partial waves, the angular amplitudes $\psi_{i}(\vec{\tau})$ describe their angular distributions, and the dynamic amplitudes $\Delta\left(m_{2 \pi}\right)$ describe the dependence of the amplitudes of the $\pi^{+} \pi^{-}$system on $m_{2 \pi}$. The production amplitudes are free parameters in a likelihood fit to the measured $\vec{\tau}$ distribution and the angular amplitudes are fully determined by the appearing angular-momentum quantum numbers. However, the dynamic amplitudes are not determined by first principle, but are a necessary model input; for the most simple case of isolated $\pi^{+} \pi^{-}$resonances, Breit-Wigner amplitudes are commonly used. Due to computational reasons, the dynamic amplitudes cannot have any free fit parameters.

To avoid the necessity for this model input in the freed-isobar approach, the dynamic amplitudes are replaced by sets of step-like functions, that approximate the dynamic amplitude by a set of constants $\alpha^{b}$ within narrow bins $b$ of $m_{2 \pi}$ [2]:

$$
\Delta_{i} \rightarrow \sum_{b \in \text { bins }} \alpha_{i}^{b} \Delta^{b}\left(m_{2 \pi}\right), \quad \text { where } \Delta^{b}\left(m_{2 \pi}\right)= \begin{cases}1 & \text { if } m_{2 \pi} \text { in } b \\ 0 & \text { otherwise }\end{cases}
$$

In this approach, the step-like functions appear like "normal" partial waves in the fit. This allows us to use the same likelihood method as in the conventional PWA and determine the dynamic isobar amplitudes encoded by the free parameters $\alpha^{b}$ directly from the data.

Such a set of steplike functions will be denoted will be denoted by $[\pi \pi]_{J_{\xi}^{P C}}$, where $J_{\xi}^{P C}$ are the $J^{P C}$ quantum numbers of the freed isobar. Partial waves with steplike dynamic isobar amplitudes will be called freed-isobar waves, waves with fixed parameterizations of the dynamic isobar amplitudes will be called fixed-isobar waves. Within the freed-isobar approach, models with any combination of freed- and fixed-isobar waves are possible.

\section{Freed-isobar PWA by COMPASS}

A first freed-isobar analysis based on an extensive PWA of a data set of $46 \times 10^{6}$ events for the process $\pi^{-} p \rightarrow \pi^{-} \pi^{+} \pi^{-} p$ collected by COMPASS in 2008 was published in ref. [1] using a set of 
88 waves. In this first analysis, seven fixed-isobar waves are replaced by three freed-isobar ones, due to matching angular momentum quantum numbers:

$$
\begin{array}{ll}
0^{-+} 0^{+}[\pi \pi]_{\mathrm{S}} \pi \mathrm{S}, 0^{-+} 0^{+} \mathrm{f}_{0}(980) \pi \mathrm{S}, 0^{-+} 0^{+} \mathrm{f}_{0}(1500) \pi \mathrm{S} & \rightarrow 0^{-+} 0^{+}[\pi \pi]_{0^{++}} \pi \mathrm{S} \\
1^{++} 0^{+}[\pi \pi]_{\mathrm{S}} \pi \mathrm{P}, 1^{++} 0^{+} \mathrm{f}_{0}(980) \pi \mathrm{P} & \rightarrow 1^{++} 0^{+}[\pi \pi]_{0^{++}} \pi \mathrm{P} \\
2^{-+} 0^{+}[\pi \pi]_{\mathrm{S}} \pi \mathrm{D}, 2^{-+} 0^{+} \mathrm{f}_{0}(980) \pi \mathrm{D} & \rightarrow 2^{-+} 0^{+}[\pi \pi]_{0^{++}} \pi \mathrm{D}
\end{array}
$$

This first study yielded very promising results, that helped understand the $[\pi \pi]_{0^{++}}$wave and to eliminate the possibility that the $\mathrm{a}_{1}(1420)$ resonance-like signal, first observed by COMPASS in ref. [3], is an artifact of the parameterization of the $\mathrm{f}_{0}(980)$ dynamic isobar amplitude. Inspired by these results, we made attempts to extend the freed-isobar approach to a larger set of partial waves.

\section{Ambiguities in the freed-isobar approach}

With increasing number of freed-isobar waves, the flexibility of the analysis model drastically increases, since one complex-valued free parameter appears for every $m_{2 \pi}$ bin of every freed-isobar wave. For some combination of freed waves, this flexibility allows to approximate continuous ambiguities in the analysis model, that have to be identified an resolved. Such ambiguities will be called zero modes in this article. A more detailed discussion of zero modes can be found in ref. [4].

\subsection{Appearance of zero modes}

Since in the freed-isobar approach, the dynamic isobar amplitudes of freed waves can take any shape — up to the finite $m_{2 \pi}$ bin width - the zero modes correspond to specific shapes $\Delta_{i}^{0}\left(m_{2 \pi}\right)$ of these dynamic amplitudes, that cancel exactly to zero at evert point $\vec{\tau}$ in phase space:

$$
\sum_{i \in \text { waves }} \psi_{i}(\vec{\tau}) \Delta_{i}^{0}\left(m_{2 \pi}\right)+\text { Bose symm. }=0,
$$

where the sum runs over all waves that are affected by the particular zero mode. Since the cancellation is exact, a shift of the isobar dynamic amplitudes $\Delta_{i}\left(m_{2 \pi}\right)$ by these shapes $\Delta_{i}^{0}\left(m_{2 \pi}\right)$ does not change the overall amplitude and thus the intensity. The $\Delta_{i}^{0}\left(m_{2 \pi}\right)$ constitute a continuous ambiguity in a freed-isobar analysis:

$$
\mid \sum_{i \in \text { waves }} \psi_{i}(\vec{\tau}) \Delta_{i}\left(m_{2 \pi}\right)+\text { Bose symm. }\left.\right|^{2}=\mid \sum_{i \in \text { waves }} \psi_{i}(\vec{\tau})\left[\Delta_{i}\left(m_{2 \pi}\right)+\mathscr{C} \Delta_{i}^{0}\left(m_{2 \pi}\right)\right]+\text { Bose symm. }\left.\right|^{2} .
$$

This ambiguity is represented by the arbitrary complex-valued coefficient $\mathscr{C}$.

\subsection{Resolution of zero modes}

The presence of zero modes may affect the result of a freed-isobar PWA and has to be accounted for, since the extracted dynamic isobar amplitudes $\alpha^{\text {fit, } b}$ can be shifted away from the physical ones $\alpha^{\text {phys, } b \text { : }}$

$$
\alpha_{i}^{\text {phys }, b}=\alpha_{i}^{\text {fit }, b}+\mathscr{C} \alpha_{i}^{0, b}
$$

where $\alpha_{i}^{0, b}$ is the shape $\Delta_{i}^{0}\left(m_{2 \pi}\right)$ of the zero mode for wave $i$ evaluated at the $m_{2 \pi}$ bin $b$. 
To remove the effect of the zero mode, we have to determine $\mathscr{C}$ such, that the resulting combination $\alpha_{i}^{\mathrm{fit}, b}+\mathscr{C} \alpha_{i}^{0, b}$ resembles the physical solution. This is done, by performing a second fit step, where the following $\chi^{2}$ function is minimized:

$$
\chi^{2}(\mathscr{C})=\sum_{i, b} \sum_{j, c} \delta_{i}^{b}(\mathscr{C})\left(\mathbf{C}^{-1}\right)_{i, j}^{b, c} \delta_{j}^{c}(\mathscr{C})
$$

where $\mathbf{C}_{i, j}^{b, c}$ is the covariance matrix of the $\alpha_{i}^{\text {fit }, b}$ obtained by the minimizing algorithm and the differences $\delta_{i}^{b}(\mathscr{C})$ are given by:

$$
\delta_{i}^{b}(\mathscr{C})=\alpha_{i}^{\mathrm{fit}, b}+\mathscr{C} \alpha_{i}^{0, b}-\alpha_{i}^{\text {model }, b} .
$$

The values $\alpha_{i}^{\text {model }, b}$ are the values of a model for the dynamic isobar amplitude of wave $i$ evaluated at the corresponding $m_{2 \pi}$ bin. Such a model can, for example, be a Breit-Wigner amplitude, if the corresponding wave is known to be dominated by a single resonance, as is true for the $\rho(770)$ waves with $[\pi \pi]_{1^{--}}$isobar, or for the $f_{2}(1270)$ isobar for waves with $[\pi \pi]_{2^{++}}$isobar.

This second fit adjusts only one complex-valued parameter, while the freed-isobar fit extracts a complex-valued parameter for every $m_{2 \pi}$ bin. Therefore, most of the information obtained with the freed-isobar method is kept, even in this second fit step is performed. To demonstrate, that resolving zero-mode ambiguities cannot change the resulting dynamic isobar amplitudes arbitrarily, we generated a Monte Carlo data set, analyzed it with the freed-isobar method, and resolved the zero-mode ambiguities using a Breit-Wigner parameterization with three different values for mass and width as constraints. The results of this study are shown in fig. 1. It can be seen, that the resulting position of the $\rho(770)$ peak (blue points) is nearly independent of the chosen constraint (gray line) and that using the correct constraint yields the Monte Carlo input shape.
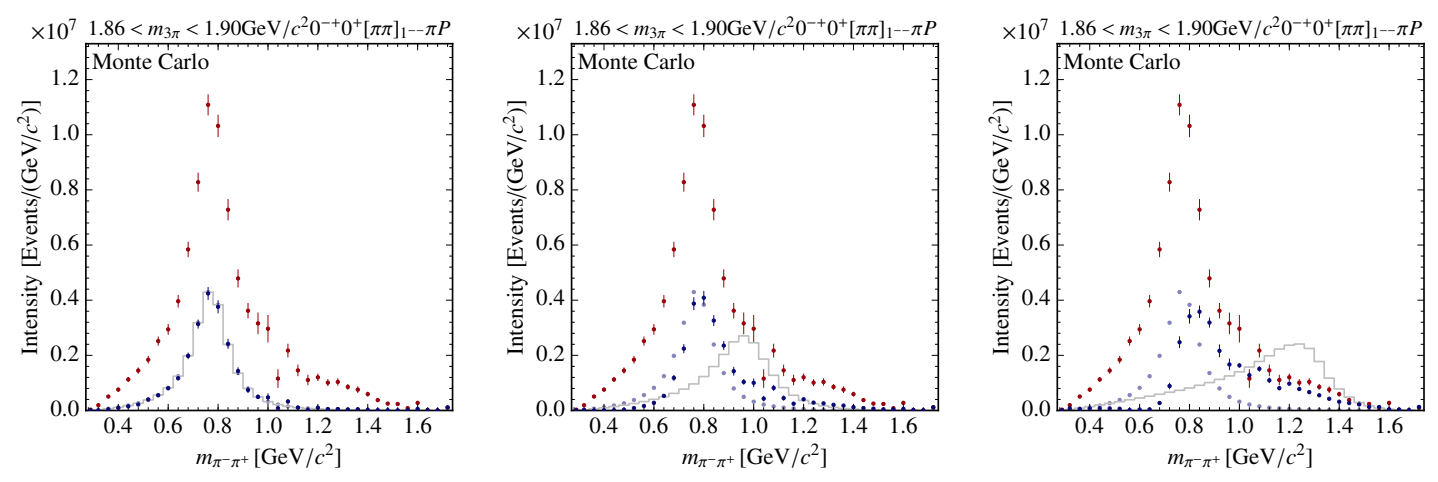

Figure 1: Results of the freed-isobar fit before (red) and after resolving of the zero mode ambiguity (blue) with different constraints (gray line). The shape used to generate the Monte Carlo data is indicated by light blue points.

\subsection{Example: spin-exotic wave}

One example for the appearance of a zero mode is the freed spin-exotic $1^{-+} 1^{+}[\pi \pi]_{1^{--}} \pi \mathrm{P}$ wave. In this case, the zero mode is contained within this single wave. Therefore, the shape of the zero-mode has to fulfill:

$$
\psi_{1^{-+}}(\vec{\tau}) \Delta_{1^{-+}}^{0}\left(m_{2 \pi}\right)+\text { Bose symm. }=0
$$


The angular amplitude for the spin-exotic wave is given by:

$$
\psi_{1^{-+}}(\vec{\tau}) \propto \vec{p}_{1} \times \vec{p}_{3}
$$

where $\vec{p}_{1,3}$ are the three-momenta of the two $\pi^{-}$in the final state in the rest frame of the three-pion system. Inserting this into eq. (3.5) and using the antisymmetry of the cross product, we obtain:

$$
0=\Delta_{1^{-+}}^{0}\left(m_{2 \pi}\right) \vec{p}_{1} \times \vec{p}_{3}+\Delta_{1^{-+}}^{0}\left(m_{2 \pi}^{1 \leftrightarrow 3}\right) \vec{p}_{3} \times \vec{p}_{1}=\vec{p}_{1} \times \vec{p}_{3}\left[\Delta_{1^{-+}}^{0}\left(m_{2 \pi}\right)-\Delta_{1^{-+}}^{0}\left(m_{2 \pi}^{1 \leftrightarrow 3}\right)\right],
$$

where $m_{2 \pi}^{1 \leftrightarrow 3}$ is the Bose symmetrized invariant two-pion mass with final-state particles 1 and 3 interchanged. Equation (3.7) is fulfilled, if $\Delta^{0}\left(m_{2 \pi}\right)$ is a constant, which is the shape of the zero mode in this wave.

To resolve the ambiguity caused by this zero mode we use the method introduced in section 3.2, using a Breit-Wigner parameterization with fixed mass and width for the $\rho(770)$ resonance as constraint. To check consistency we try a second approach, where we leave the mass and width of this parameterization as additional free parameters in the minimization of eq. (3.3). As final value for $\mathscr{C}$, we use the weighted average of both approaches.

\section{Extended freed-isobar PWA}

Based on the first freed-isobar analysis published in ref. [1], we performed an extended freedisobar analysis with twelve freed waves:

$$
\begin{array}{llll}
0^{-+} 0^{+}[\pi \pi]_{0^{++}} \pi \mathrm{S} & 1^{++} 0^{+}[\pi \pi]_{1^{--}} \pi \mathrm{S} & 2^{-+} 0^{+}[\pi \pi]_{0^{++}} \pi \mathrm{D} & 2^{-+} 0^{+}[\pi \pi]_{2^{++}} \pi \mathrm{S} \\
0^{-+} 0^{+}[\pi \pi]_{1^{--}} \pi \mathrm{P} & 1^{++} 1^{+}[\pi \pi]_{1^{--}} \pi \mathrm{S} & 2^{-+} 0^{+}[\pi \pi]_{1^{--}} \pi \mathrm{P} & 2^{-+} 1^{+}[\pi \pi]_{1^{--}} \pi \mathrm{P} \\
1^{++} 0^{+}[\pi \pi]_{0^{++}} \pi \mathrm{P} & 1^{-+} 1^{+}[\pi \pi]_{1^{--}} \pi \mathrm{P} & 2^{-+} 0^{+}[\pi \pi]_{1^{--}} \pi \mathrm{F} & 2^{++} 1^{+}[\pi \pi]_{1^{--}} \pi \mathrm{D}
\end{array}
$$

To minimize potential leakage effects 11 of the freed waves were chosen to be the eleven waves with the larges intensity in the conventional analysis in ref. [1]. In addition, the spin-exotic $1^{-+} 1^{+} \rho(770) \pi \mathrm{P}$ wave was freed, since it is a wave of major interest in the analysis. With this choice of freed waves, the analysis model has 12 fixed- and 72 freed-isobar waves. Due to the high flexibility of this model, five zero modes appear: One in the $J^{P C} M^{\varepsilon}=0^{-+}$sector, one in the $1^{++} 0^{+}$ sector, two in the $2^{-+} 0^{+}$sector, and one on the spin-exotic wave, as discussed in section 3.3. In this article, we only show results for the spin-exotic wave, therefore only the zero mode in this wave has to be resolved, which we do as described in section 3.3.

The result for the dynamic isobar amplitude for the spin-exotic wave is shown in fig. 2. The resulting dynamic isobar amplitude is dominated by the $\rho$ (770) resonance, which justifies the use of this resonance as constraint for resolving the zero-mode ambiguity. This is also supported by the fact, that resolving the ambiguity with floating $\rho(770)$ mass and width yields compatible results. These are shown in light blue in fig. 2.

Even though the dynamic isobar amplitude is dominated by the $\rho(770)$ resonance, we find, that the exact shape differs significantly from a pure Breit-Wigner shape, since the low mass tail rises slower and the high mass tail falls slower, than a pure Breit-Wigner amplitude. Reasons for this behavior can be rescattering effects with the third pion in the final state or non-resonant contributions to the process. 

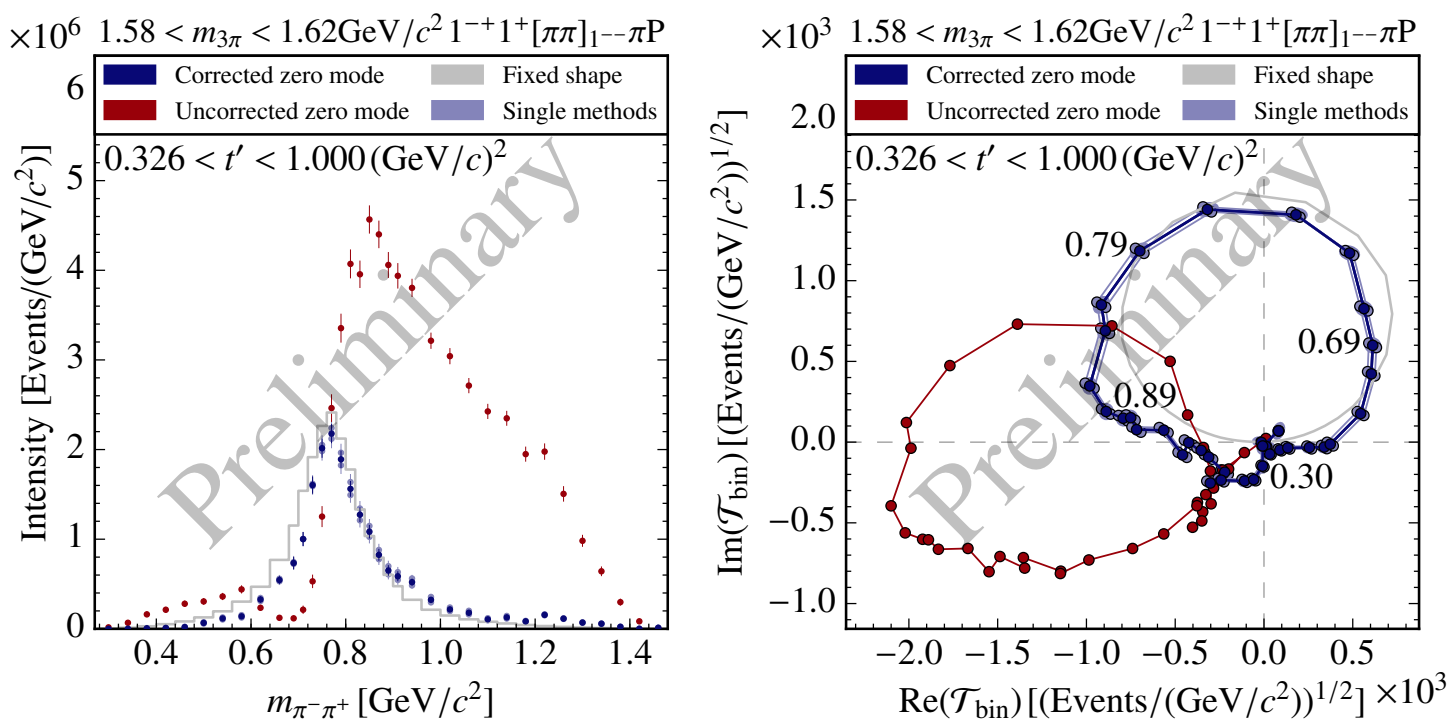

Figure 2: Intensity distribution and Argand diagram for the dynamic-isobar amplitude for the $1^{-+} 1^{+}[\pi \pi]_{1^{--}} \pi \mathrm{P}$ wave in a bin of the three-pion mass and the squared four momentum transfer $t^{\prime}$.

\section{Conclusions and outlook}

We performed a freed-isobar analysis of COMPASS data for the $\pi^{-} p \rightarrow \pi^{-} \pi^{+} \pi^{-} p$ channel, based in the analysis published in ref. [1], using an extended set of 12 freed waves. In this analysis model, we identified continuous ambiguities_zero modes—and showed a way to resolve them using additional constraints on the dynamic isobar amplitudes. With this method, we were able to extract the dynamic isobar amplitude for the spin-exotic $1^{-+} 1^{+}[\pi \pi]_{1^{--}} \pi \mathrm{P}$ wave from the data, shown in fig. 2. Even though this dynamic isobar amplitude is dominated by the $\rho(770)$ resonance, it shows significant deviations from a simple Breit-Wigner shape.

As next steps, we will extend the analysis to all bins in the three-pion mass and the squared four-momentum transfer. We will also free the dynamic isobar amplitudes of other interesting waves, for example in the $J^{P C}=3^{++}$or $4^{++}$sectors.

\section{References}

[1] COMPASS collaboration, Phys. Rev. D95 (2017), 032004.

[2] E791 Collaboration, E. M. Aitala et al., Phys. Rev. D73 (2006), 032004. [Erratum: Phys. Rev. D74, 059901 (2006)].

[3] COMPASS collaboration, Phys. Rev. Lett. 115 (2015), 082001.

[4] F. Krinner, D. Greenwald, D. Ryabchikov, B. Grube, S. Paul, arXiv:1710.09849 [hep-ph]. 